\title{
PRIMARY TUMOURS OF THE LIVER IN INFANTS AND CHILDREN*
}

\author{
BY

\begin{abstract}
H. WILliAM CLATWORTHY, JR., E. THOMAS BOLES, JR. and WILLIAM A. NEWTON Children's Hospital
\end{abstract} \\ From the Departments of Surgery and Pathology, the Ohio State University Medical School and the Columbus
}

Primary liver tumours occur in infants and children with sufficient frequency to justify careful evaluation of current techniques in diagnosis and management and to review the pathological classification of this group of neoplasms. In the past nine years (1950-1958) 15 such cases have been diagnosed at the Columbus Children's Hospital, all presenting as clinical problems; an analysis of these cases forms the substance of this report. During the same period of time 46 cases of neuroblastoma and 17 cases of embryoma of the kidney (Wilms' tumour) have been treated at this institution.

Although the treatment of liver tumours, as judged from a résumé of individual case reports in recent years, has been encouraging, the overall results achieved in significant series have been disappointing and have perhaps fostered a generally hopeless outlook for young patients with such lesions (Donovan and Santulli, 1946; Wangensteen, 1951; Lortat-Jacob and Robert, 1952; Quattlebaum, 1953; Brunschwig, 1953; Pack and Baker, 1953; Lorimer, 1955; Clatworthy and Boles, 1956). In the series from Babies' Hospital in New York City analysed by Andersen (1951) there was only one long-term survivor in 12 cases occurring over a 15-year period. This patient remained well over a six-year follow-up period after excision of the left lobe of the liver had been performed for a mesenchymoma. Gross, in a review at the Boston Children's Hospital, reports two apparent cures out of 18 such cases. One of these received radiotherapy for multiple haemangiomas and has been in excellent health for seven years thereafter; the second has been well over a 10-year period after having a wedge resection of a small haemartoma.

Safe techniques for the resection of the left lobe of the liver have been available for many years, and

\footnotetext{
* A paper read at a meeting of the British Association of Paediatric Surgeons held in Liverpool in June, 1959.
}

there are numerous reported successful cases of this procedure applied to removal of neoplasms confined to this lobe. Most of these were performed on adult patients, but a number were in children. More recently the technique for total resection of the right lobe of the liver was evolved, whereby the dangers of haemorrhage, air embolism, bile peritonitis and subsequent liver failure are minimized (Wangensteen, 1951; Lortat-Jacob and Robert, 1952; Brunschwig, 1953; Pack and Baker, 1953; Quattlebaum, 1953; Lorimer, 1955). The application of one or the other of these procedures should permit the surgical excision of most liver tumours which are not centrally placed or diffuse or multicentric lesions.

\section{Diagnosis}

The vast majority of primary neoplasms of the liver produce symptoms in the first two years of life. Only two of the 15 cases in this series had the onset of symptoms after 2 years of age, and in three instances the clinical manifestations were present in the neonatal period.

Abdominal enlargement due to a palpable abdominal mass is by far the most common presenting complaint. The distribution of these tumours in the liver, and hence the location of the mass, seems to follow the distribution of the liver mass. Of these 15 tumours, nine were in the right lobe, three in the left and two were situated centrally. One was diffuse. In most instances the mass was in the right upper quadrant, and less often in the mid-epigastrium. The mass moves with respirations. Its characteristics on palpation vary considerably, but most tumours are hard and rounded and inseparable from the liver edge. Transillumination is a useful tool in diagnosis since a liver tumour which transilluminates is almost certainly a large lymphangioma.

Other clinical manifestations observed in this 
series include anorexia, fever, weight loss and rarely jaundice. This latter sign was observed in only a single case in this series, a 14-year-old girl who died from a liver cell carcinoma associated with postnecrotic cirrhosis. In a single instance, heart failure in a newborn infant progressed to death at 8 weeks, with the pathological findings of diffuse haemangiomas in the liver, skin and pancreas, as has been previously described by Winters, Robinson and Bates (1954) and by Levick and Rubie (1953).

Roentgenological studies are useful in distinguishing hepatic tumours from embryomas of the kidney and neuroblastomas. An intravenous pyelogram is particularly helpful. The kidneys function normally and are usually in normal position in right-sided tumours, although the pelvis and calices may be compressed. Distortion of the pelvis and calices, as seen with a Wilms' tumour, is not present, nor is lateral displacement of the kidney, as with a neuroblastoma, common. The mottled calcification so characteristic of neuroblastoma was not observed, although calcification has been described in some liver tumours. Barium studies of the gastrointestinal tract show displacement of these viscera away from the tumour as one would expect. Downward displacement of the duodenum and hepatic flexure of the colon is seen with neoplasms of the right lobe, in contrast to the forward or medial displacement usually seen with a renal embryoma or neuroblastoma.

Laboratory tests have been of little diagnostic value in these cases. Liver function studies were all essentially normal except in the case of the child with diffuse post-necrotic cirrhosis. Abnormalities in the liver function tests should lead one to question the diagnosis of primary liver tumour. For example, a diagnostic error was made in a newborn infant, admitted at 2 days of age with jaundice and a large epigastric mass. The jaundice persisted as well as other laboratory evidence of disturbed liver function. At operation at 3 weeks of age the mass proved to be a hard, nodular, grey-white 'tumour' occupying the left lobe. Resection of the left lobe was performed and the child recovered without difficulty. However, on microscopical examination this proved to be a large cirrhotic nodule possibly associated with post-intrauterine hepatitis, with marked fibrous tissue proliferation, replacing the normal architecture of the hepatic tissue. The hepatic cells were completely or partially destroyed and showed irregular arrangement. The bile ducts appeared to be increased. It is interesting that the jaundice disappeared within a month after operation and the infant has been perfectly well over a subsequent eight-month period.

\section{Classification}

The wide variety of gross and microscopical characteristics of primary liver tumours and the relatively small number of such cases collected at any one institution have made the adoption of a uniform classification difficult (Warvi, 1944; Edmondson, 1956). To add to the problem is the fact that the clinical behaviour is not always correlated with the microscopical appearance, so that a classification based on the pathological data alone appears to be unsatisfactory. For practical reasons it therefore seems appropriate to group these tumours in three broad categories:

1. Tumours of Hepatic Cell Origin. This group is made up of all tumours derived from entodermal cells and includes hepatomas, cholangiomas and the cholangiohepatomas. There were no adenomas in this series and the hepatomas have invariably behaved as malignant lesions, although the histological appearance may suggest benignity (Fig. 1a and b).

2. Tumours of Supporting Structures. This group comprises those tumours of mesodermal origin including fibromas, haemangiomas, lymphangiomas, fibroangiomas and mesenchymomas. Such neoplasms may be benign, as are most of those in this series, or malignant.

3. Mixed Tumours and Teratomas. Such tumours contain cells derived from more than one germ layer. Osteoid tissue, for example, may be seen in liver cell tumours. Teratomas are distinctly rare (Fig. 2).

In this series hepatoma was the most common tumour encountered, occurring in 10 cases. Five were in the right lobe, two in the left and two were centrally positioned. In eight cases symptoms developed in the first two years of life, and the ninth patient had his initial symptoms at 2 years 11 months of age. None of these nine patients had associated cirrhosis so commonly found in adult cases. However, as already mentioned, the tenth case occurred in a 14-year-old girl with post-necrotic cirrhosis of the liver. There were no instances of adenomas or exclusively bile duct tumours among these cases and only a single case of histologically benign cholangiohepatoma occurring in the right lobe of a 10-weekold infant.

Among the tumours of supporting tissue origin were one diffuse haemangioma, two lymphangiomas and one fibroangioma. There were no mesenchymomas, mixed tumours or teratomas. Both 


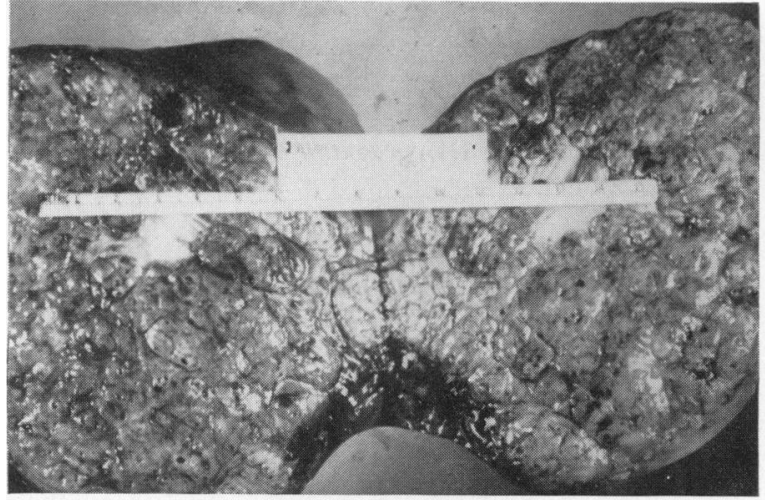

FIG. 1 (a).-Gross and microscopical appearances of a well-differentiated hepatoma of left lobe of liver successfully excised from a 15-month-old infant who subsequently died of undifferentiated hepatic and pulmonary metastases seven months after original resection.
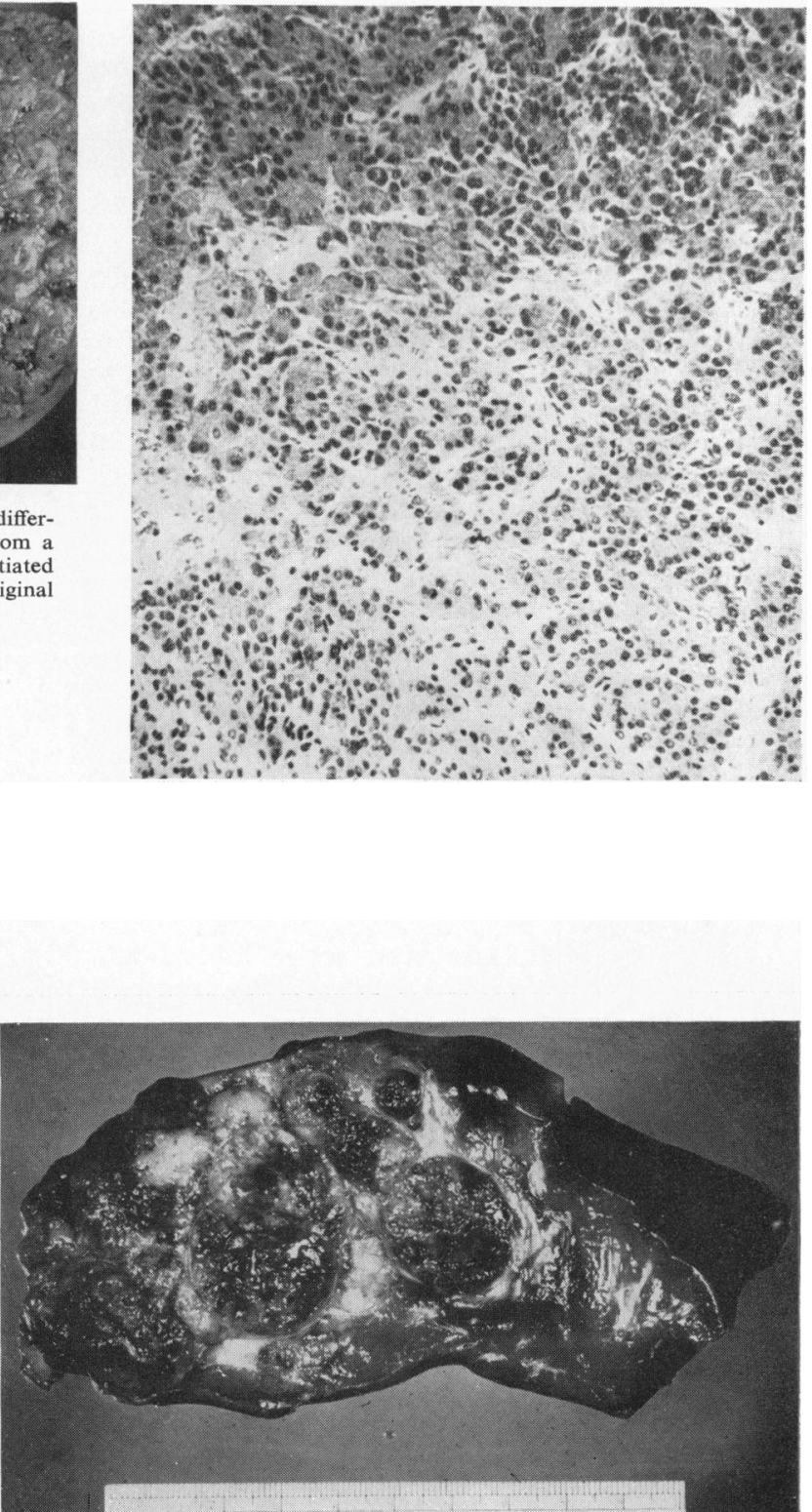

FIG. 1 (b).-Gross and microscopical appearances of a very rapidly growing anaplastic hepatoma of left lobe of liver easily resected with wide margin of normal liver substance in a 2-year-old infant who succumbed to multiple recurrences in right lobe of liver within five months. 

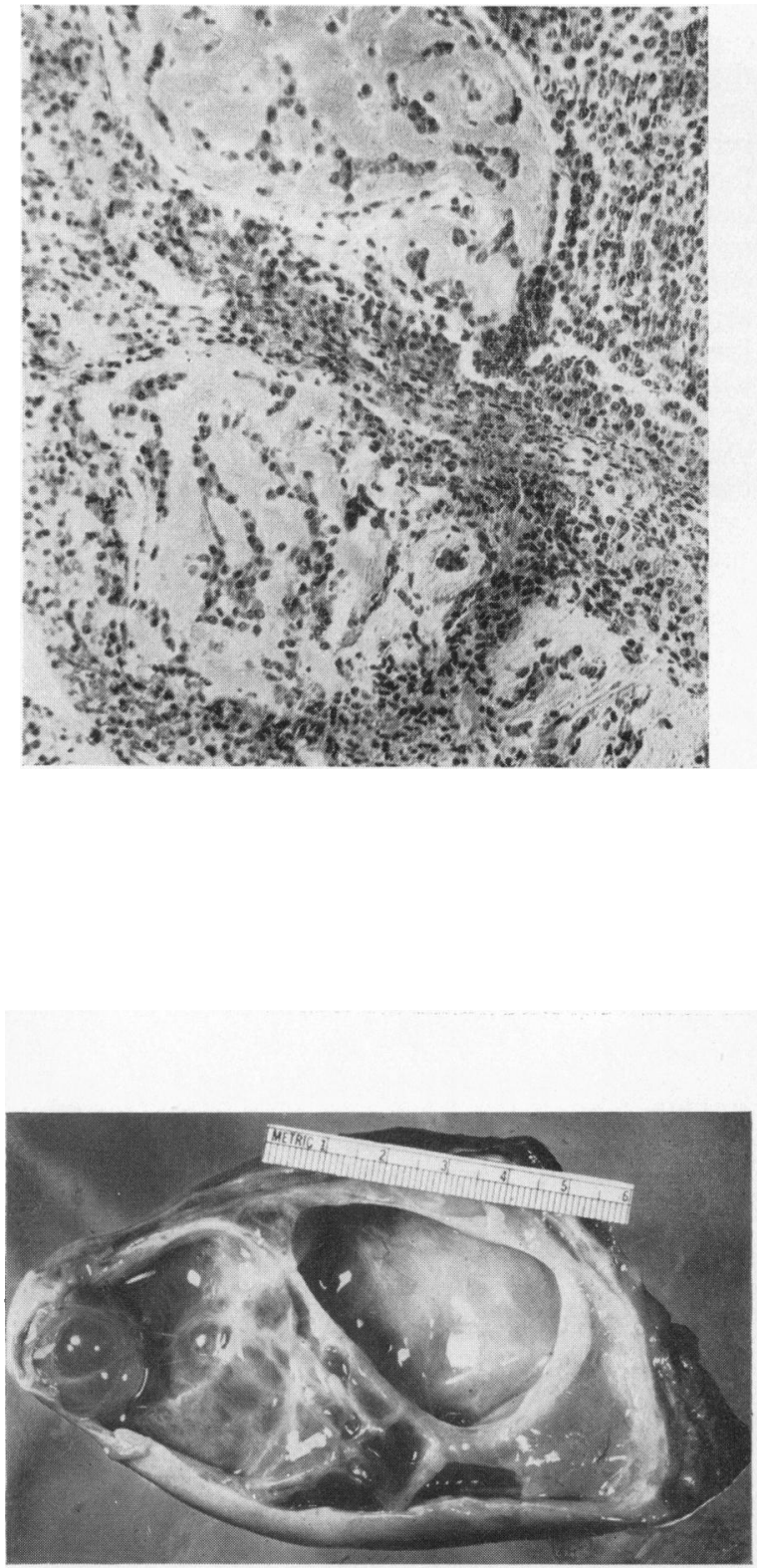

FIG. 3.-Gross and microscopical appearances of a huge rapidly growing lymphangioma involving entire right lobe of liver which was successfully excised from a 9-month-old infant who is well nine months later.

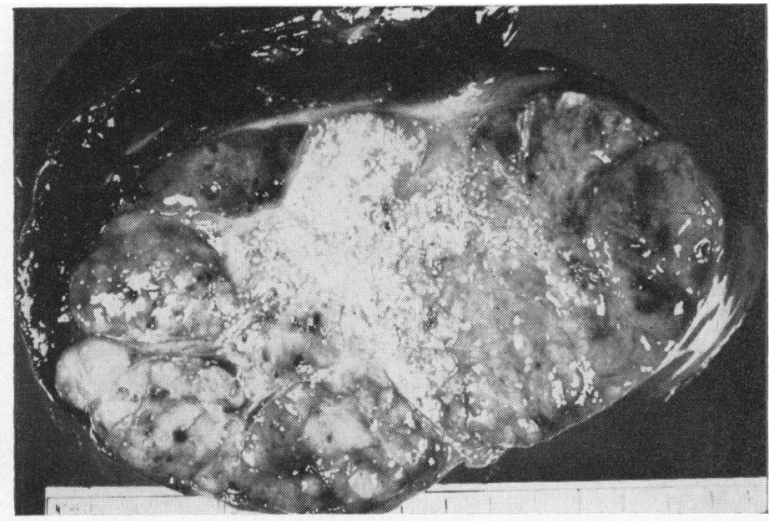

FIG. 2.-Gross and microscopical appearances of mixed tumour of liver in a young child; it contained highly undifferentiated hepatoma as well as areas of osteoid tissue.

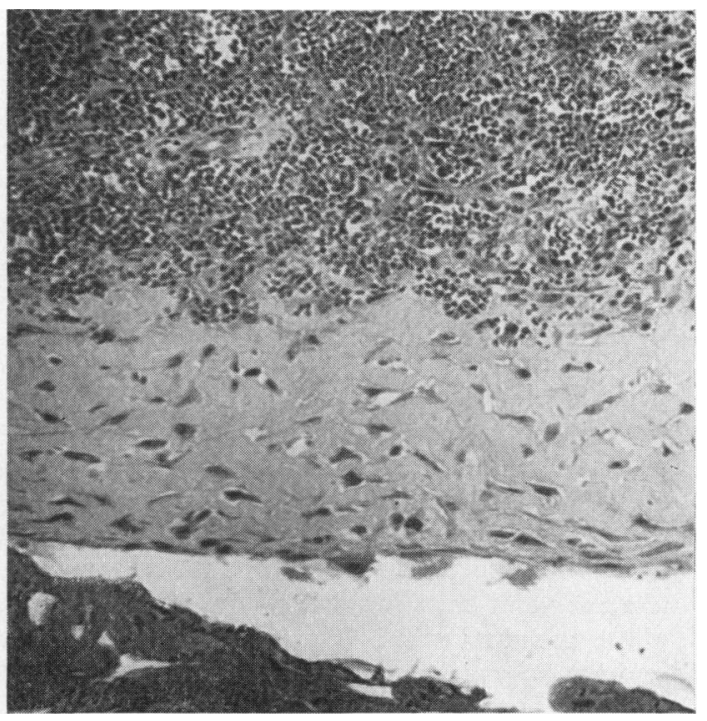


lymphangiomas were found in the right lobe of infants, one $4 \frac{1}{2}$ months and the other 9 months of age (Fig. 3). The patient with diffuse haemangiomatosis, already noted, had symptoms at birth. The liver of this patient had numerous small capillary haemangiomas scattered diffusely throughout all portions of the liver parenchyma. The single case of fibroangioma was a newborn infant with the neoplasm originating in the left lobe.

There is a strikingly similar gross appearance of many of these tumours, so that differentiation by this means alone is often quite unreliable. Similarly, the differentiation microscopically of benign and malignant liver cell tumours is occasionally virtually impossible. In one of the hepatomas in this series the cytology and architecture were most suggestive of a benign neoplasm, but the presence of metastases established the malignant nature of the tumour.

\section{Treatment}

Effective treatment of primary hepatic neoplasms is at present limited almost entirely to surgical resection. Radiotherapy may be of value in haemangiomas, as suggested by Gross, and in one of our cases of inoperable hepatoma radiotherapy resulted in definite regression in size of the tumour mass. Systemic chemotherapy has not been of value to date.

In tumours limited to either the right or left lobe, surgical removal by total right or left lobectomy is a practical procedure. Such procedures take advantage of the anatomical division of the organ into right and left lobes, each with a largely separate blood supply from the hepatic artery and portal vein. Control of the blood supply and the bile duct system at the hilum of the liver plus similar control of the hepatic veins draining into the inferior vena cava make possible such resections without excessive risk from haemorrhage, air embolism, or bile peritonitis.

In such a procedure for a tumour involving the right lobe the diagnosis is confirmed and operability determined through an abdominal incision. If resection appears possible the abdominal incision is joined or extended by an incision into the right chest through the seventh or eighth intercostal space, and the right diaphragm is divided down to the vena cava. Umbilical tapes are then placed around the inferior vena cava just below the right atrium and above the renal veins. The hilar structures are freed and the appropriate branches of the hepatic artery, portal vein and hepatic duct are carefully identified, ligated and divided. The short hepatic veins from the right lobe to the vena cava are next ligated and divided. This is the most dangerous part of the procedure since extension of the tumour into this area may not be appreciated until this part of the dissection is under way, and if such extension is encountered there is a considerable risk of haemorrhage or air embolism. The previously placed tapes on the vena cava and control of the hilar structures permit control of such a situation. Following this the liver is transected to the right of the falciform ligament down to the vena cava. During the actual transection the hilar structures are cross-clamped with a Pott's clamp. Interruption of the blood supply by this means can safely be tolerated by the liver for about 15 minutes, and probably longer with the controlled moderate hypothermia $\left(90^{\circ}\right.$ $92^{\circ}$ F.) customarily employed in these cases. All visible blood vessels and bile ducts are ligated as the dissection proceeds. If the gallbladder is not involved, it may be peeled out of its bed before the transection and used after removal of the lobe for retrograde injection of a methylene blue solution. By this means additional bile ducts can be visualized and ligated. The falciform ligament and posterior peritoneum are used to reperitonealize the raw surface. If this is not sufficient, a layer of compressed Ivalon may be sutured in place to cover the remaining raw area. Penrose drains are placed to drain the right subdiaphragmatic space so that a localized collection of bile or a bile peritonitis will not occur.

The left lobe can be mobilized more easily than the right and this can ordinarily be done through an upper abdominal incision by dividing the peritoneal attachment to the diaphragm and the falciform ligament. It is quite easy to place a row of interlocking mattress sutures just to the left of the falciform ligament and resect the left lobe. It has not been found necessary to perform a hilar dissection or to occlude the hilar structures during this procedure. Again, however, reperitonealization of the raw surface and drainage are important steps.

\section{Results}

There have been no long-term survivors in the 10 patients with hepatoma. In one, the diagnosis was not made until post-mortem examination and no treatment was given. In two, only exploration and biopsy were performed, and both died within three months. In two others, exploration and biopsy were followed by radiotherapy. The tumour mass strikingly decreased in size in one of these, but none the less the patient lived only six months after completion of the treatment. The other lived only four months after therapy.

Right lobectomy was performed in four patients and there were three early deaths. One of these 
patients died from operative haemorrhage, and a second from haemorrhage and air embolism. A third unfortunate death resulted from staphylococcal peritonitis and septicaemia two weeks after operation and at autopsy no tumour was found. All three deaths must be considered preventable. The first two cases were in retrospect inoperable because of tumour invasion across the midline posteriorly in the area of the hepatic veins. The third death presumably followed contamination in the operating room or via the drain site. The fourth patient who had a resection of the right lobe died at home two and a half months after operation following a brief acute illness suggestive of intestinal obstruction. The patient was not returned to the hospital during this illness and no autopsy was performed. A left lobectomy was performed in the final patient, and he died with massive metastases in the right lobe seven months after resection. This last patient had been known by his parents to have an abdominal lump since 3 months of age, but no treatment was sought until 15 months later at which time a huge tumour in the left lobe was found.

The single patient with a cholangio-hepatoma had a resection of the right lobe of the liver at 10 weeks of age (Fig. 4). He is living and well 12 months later with no evidence of recurrence.

Both patients with giant lymphangiomas had tumours occupying almost all of the right lobe. Resections of the right lobe were done at 5 and 9 months, and both patients are in good health

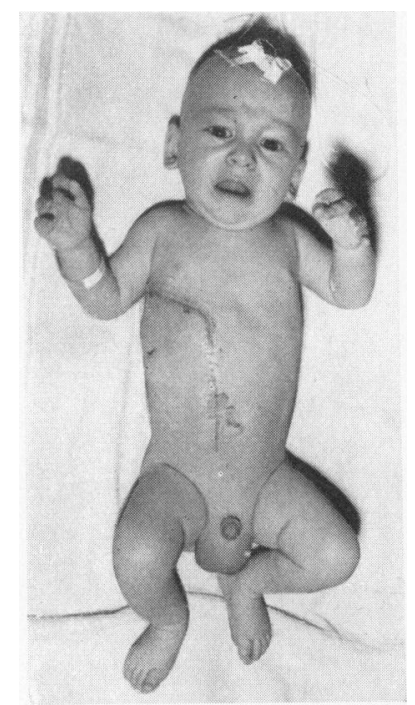

Fig. 4.-A 10-week-old infant one week after total right hepatectomy for cholangio-hepatoma.

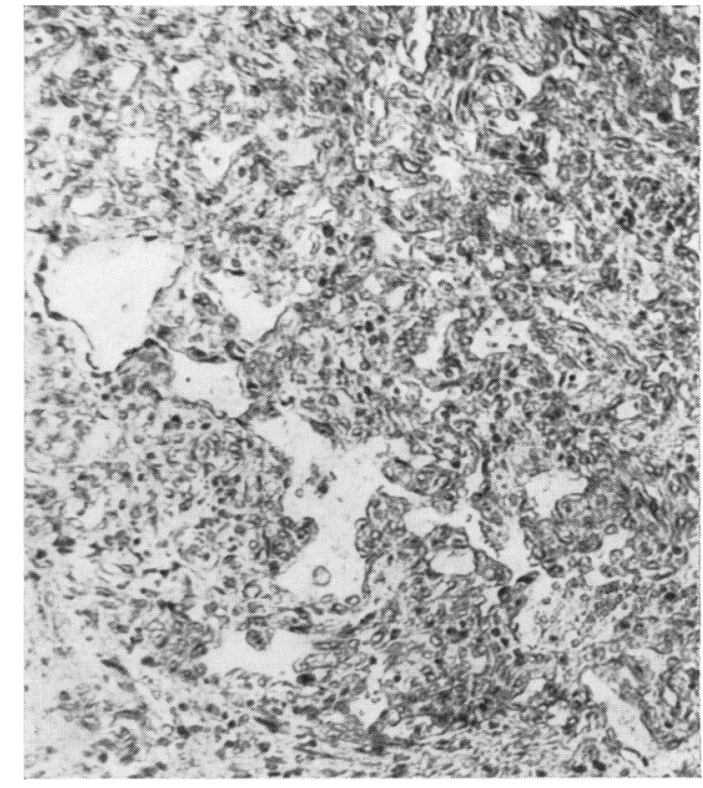

FIG. 5.-Microscopical appearance of liver removed at autopsy from an 8-week-old infant with multiple diffuse haemangiomas who had been under treatment since birth for 'heart failure'.

six and a half years and nine months later. The infant with a fibroangioma of the left lobe had a left hepatectomy at 3 weeks of age, and is well 14 months later.

None of the long-term survivors after resection of the right or left lobe shows any evidence of impairment of liver function. Although in all cases there was a plateau in weight gain for a few weeks after operation, subsequent growth and development patterns have been entirely normal.

The patient with diffuse haemangiomas of the liver, as well as of the pancreas and skin, was treated for heart failure from birth and died at 8 weeks of age. The diagnosis was made from the autopsy findings (Fig. 5).

\section{Discussion}

Primary liver tumours are not rare paediatric problems. Without treatment the prognosis is poor in both the malignant and benign varieties, as indicated by the larger reported series (Andersen, 1951; Gross, 1953; Lee, Newstedt and Siddall, 1956). With adequate surgical resection the prognosis in cases of benign tumours is good, and after such treatment normal growth and freedom from liver impairment may be expected. The outlook for patients with malignant tumours, even after operation, has thus far been poor, but with the 
development of more adequate techniques in achieving wide resections there is reason to believe that success may be achieved. One remarkable exception is the case reported by Lee et al. (1956) of an infant with a huge hepatoma confirmed by biopsy. No treatment was given but spontaneous regression occurred and the child was reported in good health 10 years later.

Establishing a clinical diagnosis of a primary liver tumour is ordinarily not difficult; however, the differentiation of a benign from a malignant tumour clinically or at operation may be quite impossible. Two such dilemmas occurred in this series. Since surgical excision currently affords the only chance of eradicating such lesions, it is our opinion that in the absence of distant metastases isolated lesions should be removed.

The techniques of resection of either the right or the left lobe are sufficiently well developed to permit their application with a reasonable assurance of success. The major problems are haemorrhage, air embolism and bile peritonitis. An anatomical technique with control of the afferent and efferent blood supply, controlled hypothermia, reperitonealization of the cut surface and adequate drainage are methods which contribute to the success of these procedures.

\section{Summary}

The majority of primary liver tumours which present as clinical problems in children are malignant and occur in the first two years of life. Such tumours develop in a normal liver, and are not superimposed on a cirrhotic process as in adults.

From the practical standpoint these neoplasms can be classified as (1) tumours derived from liver cells, (2) tumours derived from supporting cells, and (3) mixed tumours. Those derived from liver cells are malignant in the vast majority of cases, although the microscopical picture of some is quite benign.

The solid tumours of the liver should be treated in a uniform manner, adequate surgical excision through normal liver tissues being the best available procedure.

A surgical technique based on sound anatomical principles and planned to minimize the risks of haemorrhage, air embolism and bile peritonitis makes removal of these tumours by a resection of either the right or left hepatic lobes a logical and practical procedure.

\section{REFERENCES}

Andersen, Dorothy H. (1951). Tumours of Infancy and Childhood. Cancer, 4, 890.

Brunschwig, A. (1953). Surgery of Hepatic Neoplasms. Cancer, 6, 725 .

Clatworthy, H. W., Jr. and Boles, E. T. Jr. (1956). Right Lobectomy of the Liver in Children. Surgery, 39, 850.

Donovan, E. J. and Santulli, T. V. (1946). Resection of the Left Lobe of the Liver for Mesenchymoma. Ann. Surg., 124, 90.

Edmondson, H. A. (1956). Differential Diagnosis of Tumors and Tumor-like Lesions of Liver in Infancy and Childhood. A.M.A. J. Dis. Child., 91, 168.

Gross, Robert E. (1953). The Surgery of Infancy and Childhood.

p. 532. Saunders \& Co., Philadelphia.

Lee, C. M. Jr., Newstedt, J. R. and Siddall, H. S. (1956). Large Abdominal Tumors of Childhood (Other than Wilms' Tumor or Neuroblastoma). Ann. Surg., 143,803.

Levick, C. B. and Rubie, J. (1953). Haemangioendothelioma of the Liver Simulating Congenital Heart Disease in an Infant. Arch. Dis. Childh., 28, 49.

Lorimer, W. S. Jr. (1955). Right Hepatolobectomy for Primary Mesenchymoma of the Liver. Ann. Surg., 141, 246.

Lortat-Jacob, J. L. and Robert, H. G. (1952). Hépatectomie droite réglée. Presse méd., 60, 549.

Pack, G. T. and Baker, H. W. (1953). Total Right Hepatic Lobec-

tomy. Ann. Surg., 138, 253.
Quattlebaum, J. K. (1953). Massive Resection of the Liver, Ann. Surg., 137, 787.

Wangensteen, O. H. (1951). Cancer of the Esophagus and the Stomach. American Cancer Society, Inc., p. 92.

Warvi, W. N. (1944). Primary Neoplasms of the Liver. Arch. Path., $37,367$.

Winters, R. W., Robinson, S. J. and Bates, G. (1954). Hemangioma of the Liver with Heart Failure: A Case Report. Pediatrics, 14, 117. 